\title{
A review on fiber optic sensor used in electronics industry, internet of things, and new generation optical communications networks
}

\author{
F E Serajii ${ }^{1}$, M Ghanbari Sabagh ${ }^{2}$, and D Ranjbar Rafi ${ }^{2}$ \\ 1. Department of Optical Telecommunication, Institute of Communication Technology, Tehran, Iran \\ 2. Faculty of Engineering, Islamic Azad University, Tehran, Iran \\ E-mail: feseraji@itrc.ac.ir
}

\begin{abstract}
Fiber-optic sensors available in the market are used to measure physical quantities such as electrical and magnetic fields, electrical current, temperature, pressure, acceleration, flow of liquids and gases, and measurement of fluid levels in various industries. As of today, due to the growing use of fiber optics, manufacturers of many machineries and control systems have been keen on using these sensors in manufacturing products. In this paper, due to the increasing importance of optical sensors, especially in wireless sensor networks, in the Internet of Things, and in new generation optical fiber communication networks, these types of sensors have been widely studied over the past decades to date, and in terms of technical and their applicabilities, have been reviewed and reported. The results of this article can be used by designers of these sensors in various industries and scholars of physics and engineering departments at undergraduate and post-graduate level.
\end{abstract}

Keywords: optical fiber sensors, electronic industry, physical quantity, optical communication networks, IoT, WSNs.

For full article, refer to the Persian section 